\title{
Contemporary outcomes of continuous-flow biventricular assist devices
}

\author{
James Farag ${ }^{1,2}$, Kei Woldendorp ${ }^{3}$, Nicholas McNamara ${ }^{1,2,4}$, Paul G. Bannon ${ }^{1,2}$, Silvana F. Marasco, \\ Antonio Loforte ${ }^{7}$ Evgenij V. Potapov ${ }^{8,9}$ \\ ${ }^{1}$ Cardiothoracic Surgical Department, Royal Prince Alfred Hospital, Sydney, Australia; ${ }^{2}$ Baird Institute of Applied Heart and Lung Research, Sydney, \\ Australia; ${ }^{3}$ Cardiothoracic Surgical Department, Royal North Shore Hospital, Sydney, Australia; ${ }^{4}$ Faculty of Health and Medicine, University of \\ Sydney, Sydney, Australia; ${ }^{5}$ Cardiothoracic Surgical Department, The Alfred, Melbourne, Australia; ${ }^{6}$ Department of Medicine and Surgery, Monash \\ University, Melbourne, Australia; ${ }^{7}$ Division of Cardiac Surgery, IRCCS Azienda Ospedaliero-Universitaria di Bologna, Bologna, Italy; ${ }^{8}$ Deutsches \\ Zentrum für Herz Kreislauf Forschung (DZHK) - Standort Berlin/Charité, Berlin, Germany; ${ }^{9}$ Department of Cardiothoracic and Vascular Surgery, \\ Deutsches Herzzentrum Berlin (DHZB), Berlin, Germany \\ Correspondence to: James Farag. Cardiothoracic Surgical Department, Royal Prince Alfred Hospital, Sydney, Australia. Email: faragjames@gmail.com.
}

Background: Significant right ventricular failure (RVF) complicating left ventricular assist device (LVAD) placement has been reported at 10-30\%. Although primarily indicated for left ventricular failure, ventricular assist devices (VADs) have become utilized in a biventricular setup to combat right ventricular failure (RVF) following LVAD implantation. With the advent of continuous-flow LVADs (CF-LVADs) superseding their pulsatile predecessors, the shift towards CF-biventricular assist devices (CF-BiVADs) come with the prospect of improved outcomes over previous pulsatile BiVADs. We aim to review the literature and determine the outcomes of CF-BiVAD recipients.

Methods: A systematic review was performed to determine the outcomes of CF-BiVADs. Pre-operative demographics and device configuration data was collected. Primary outcomes evaluated were short-term survival, long-term survival, duration of support, and survival to transplant. Secondary outcomes evaluated included intensive care unit (ICU) and hospital length of stay (ICU-LOS and HLOS, respectively), pump thrombosis, pump exchange. Median and interquartile range was reported where appropriate. A major limitation was the likely overlap of cohorts across publications, which may have contributed to some selection bias.

Results: Of 1,282 screened, 12 publications were evaluated. Sample size ranged from 4 to 93 CF-BiVAD recipients, and follow-up ranged from 6 to 24 months. Mean age ranged from 34 to 52 years old. Forty-five percent of CF-BiVADs had right atrial (RA-) inflow cannulation, with the remaining being right ventricular (RV). Thirty-day survival was a median of 90\% (IQR 82-97.8\%) and 12-month survival was a median of $58.5 \%$ (IQR 47.5-62\%). Where reported, rate of pump thrombosis (predominantly the right VAD) was a median of $31 \%$ (IQR 14-36\%), although pump exchange was only 9\% (IQR 1.5-12.5\%).

Conclusions: RVF post-LVAD implantation is a high morbidity and mortality complication. There is no on-label continuous-flow RVAD currently available. Thus, the modifications of LVADs for right ventricular support to combat pump thrombosis has resulted in various techniques. BiVAD recipients are predominantly transplant candidates, and complications of pump thrombosis and driveline infection whilst on wait-list are of great consequence. This study demonstrates the need for an on-label CF-BiVAD.

Keywords: Biventricular assist device (BiVAD); heart failure; continuous-flow

Submitted Jan 18, 2021. Accepted for publication Mar 16, 2021.

doi: 10.21037/acs-2021-cfmcs-34

View this article at: http://dx.doi.org/10.21037/acs-2021-cfmcs-34

(C) Annals of Cardiothoracic Surgery. All rights reserved. Ann Cardiothorac Surg 2021;10(3):311-328 I http://dx.doi.org/10.21037/acs-2021-cfmcs-34 


\section{Introduction}

Heart failure refractory to medical therapy has been treated with short- and long-term mechanical circulatory support since the 1960s. The first implantations of a left ventricular assist device (LVAD) as well as the first use of extra-corporeal life support (ECLS) heralded an innovative approach to mechanical circulatory support that has transformed the approach to heart failure. These advances have significantly improved survival to transplant, and evolved into a destination therapy for those indicated (1).

Although primarily indicated for left ventricular failure (LVF), ventricular assist devices (VADs) have become utilized in a biventricular set up to combat right ventricular failure (RVF) following LVAD implantation. Significant RVF complicating LVAD placement has been reported at $10-30 \%(2,3)$, and the need for either temporary or permanent mechanical right ventricular support occurs in $6-11 \%$ of LVAD recipients $(2,4)$. Development of right heart failure following implantation of LVAD is shown to increase mortality six-fold and is a major contributor to prolonged hospitalization and re-hospitalization $(5,6)$. Multiple studies have attempted to predict those patients requiring mechanical RV support post-LVAD (6-10), though they remain inadequately validated secondary to poor sensitivity and specificity $(11,12)$.

Implantation of right-sided mechanical support can be temporary or permanent, and concomitant or delayed. Although temporary devices are aimed at avoiding the need for durable VAD implantation, they often still lead to a deferred right ventricular assist device (RVAD). Importantly, delayed implantation of permanent right ventricular mechanical devices has a significantly higher risk of mortality and morbidity than concomitant implantation (13). Survival following biventricular assist device insertion is reported to be $56 \%$ at 1 -year (14).

A permanent and on-label long-term mechanical solution for right ventricular devices does not exist outside of use of the Total Artificial Heart (TAH) and the BerlinHeart Excor-a pulsatile device that may be used as biventricular support in Europe. However, the TAH does not fall into the classification of biventricular assist device (BiVAD) as it replaces the failing ventricles as opposed to assisting them (15). Hence, without an on-label solution, clinicians have resorted to using left-sided continuous-flow ventricular assist devices (VADs) in the right sided position for patients with severe biventricular failure (16).
The transition to continuous flow (CF) devices was accelerated by results of studies demonstrating mechanical problems-thrombosis, pump failure, system membrane rupture-occurred more frequently in pulsatile devices (17). Furthermore, in a randomized control trial comparing CF to pulsatile LVADs, it was found that the overall composite end-point of 2-year survival free of disabling stroke or re-operation was significantly better in the CF group (17). Continuous-flow devices have since superseded pulsatile devices. They have been shown to have at least equal survival to transplant, but also longer duration of support (18). The first use of a durable continuousflow VAD in the right side was described in 2004, in a biventricular configuration (19) and since then, CF-BiVADs have progressively become the device configuration of choice. An INTERMACS (Interagency Registry for Mechanically Assisted Circulatory Support) report published in 2017 recorded a total of 22,866 mechanical support devices implanted from 2006-2016. Of these, 616 were continuous-flow BiVADs (CF-BiVADs) (2.7\%) and 349 pulsatile BiVADs (1.5\%) (20). The latest INTERMACS report showed that the proportion of CF-BiVADs had dramatically replaced the use of pulsatile devices $(3.9 \% v s$. $0.1 \%$, respectively), with BiVAD implantation composing $4.1 \%$ of all durable devices (21).

Without a dedicated, on-label solution for severe biventricular failure in the VAD market, there have been various ad-hoc techniques to RVAD or BiVAD implantation. Each of these have their own unique pitfalls and advantages. This review aims to evaluate the overall outcomes of BiVADs, in particular the now widely accepted continuousflow devices that have superseded pulsatile flow devices.

\section{Methods}

\section{Literature search strategy}

Six databases were used to perform electronic searches including Ovid MEDLINE, EMBASE, PubMed, Cochrane Database of Systematic Reviews (CDSR), SCOPUS and Database of Abstracts of Review of Effectiveness (DARE). These databases were searched from their dates of inception to December 2020. The search strategy included a combination of keywords and MeSH headings including biventricular assist devices OR BiVAD (Figure S1). Predefined selection criteria were used to assess all relevant articles that were identified. 


\section{Selection criteria}

Outlined below are the inclusion and exclusion criteria for our search. Long-term data was defined as 2 years or more follow-up post-operatively.

Inclusion criteria: (I) continuous flow right and left ventricular devices; (II) prospective and retrospective studies; (III) case series, meta-analyses; (IV) pre- or posttransplant patients.

Exclusion criteria: (I) non-English language titles without adequate translation; (II) pneumatic or pulsatile ventricular assist devices; (III) single ventricular assist device; (IV) paediatric population ( $<17$ years); (V) small case series $(\mathrm{n}<4)$; (VI) case reports, abstracts, conference presentations, editorials, reviews, and expert opinions.

\section{Data extraction and critical appraisal}

Patient-level data was extracted from article texts, tables and figures (JF, KW, NM). Discrepancies were discussed between reviewers and a consensus was reached.

\section{Outcomes of interest}

Primary outcomes evaluated were short- and long-term survival, duration of support, and survival to transplant. Secondary outcomes assessed were complications including pump thrombosis, pump exchange, bleeding, stroke, infection, and intensive care unit (ICU) and hospital length of stay.

\section{Statistical analysis}

Due to the limited numbers of BiVAD recipients, likelihood of duplication, and varying end-points measured, a metaanalysis was not able to be performed. Results are presented comparing demographics, methods of BiVAD implantation, survival, and complications. Where data is sufficiently reported, means and medians are provided to assess overall outcomes.

\section{Results}

\section{Quality of studies}

The literature search identified a total of 1,612 studies. After exclusion of duplicates and irrelevant studies, 59 publications were selected for full-text review. Fourteen studies with a total of $399 \mathrm{CF}-\mathrm{BiVAD}$ recipients were reviewed. One study was a systematic review (16), and all others were observational studies. Three studies were registry reviews, one Interagency Registry for Mechanically Assisted Circulatory Support (INTERMACS), one European Registry of patients with Mechanically Assisted Circulatory Support (EUROMACS), and one of the United Network for Organ Sharing (UNOS) database. A further three studies were multi-center observational studies and the remaining seven publications were case series. All studies were retrospective reviews of prospectively collected data. Reported follow-up duration ranged from a mean of four to twenty-one months. Most studies, however, did not report a mean/median follow-up period. Number of participants ranged from 4 to 93 receiving continuousflow BiVADs. Although multiple studies included a greater number of BiVAD recipients, all but one (22) did not report on outcomes of CF-BiVADs separately, and thus were not included in the analysis. Quality assessment of each study was performed using the GRADE system and can be seen in Table 1.

\section{Basic demographics and pre-operative status}

Mean age ranged from 34 to 52 years old (Table 2), and gender was predominantly male $(74-100 \%)$. Where reported (11 of 14 papers), BiVAD recipients were primarily implanted for bridge to transplant (BTT), ranging from $47-100 \%$, except in one study, where BTT was only $27 \%$ of recipients. In this study, bridge to candidacy/decision was also $27 \%$ (23). INTERMACS profile-which characterizes severity of cardiogenic shock (Figure 1)—demonstrated that most BiVAD recipients were critically unwell with $65-100 \%$ of recipients INTERMACS 1 or 2 . A median of $24 \%$ (IQR 11-34\%) patients were bridged from ECMO (9/14 studies reported). Non-ischaemic cardiomyopathies were the predominant etiology, with dilated cardiomyopathy being the most commonly reported subtype (37-92\%).

\section{Devices, techniques and configurations}

Although device numbers were not consistently reported, most studies delineated which devices were utilized on their participants (Table 3). Eleven of these studies (15,16,23-31) utilized the HeartWare HVAD (Medtronic, Minnesota $\mathrm{MN}$ ) whereas only three studies reported the use of the HeartMate 3 (Abbott, Chicago, IL) (27,32,33) (Figure 2). Timing of RVAD implantation as either concomitant with LVAD versus delayed was reported in most studies 
Table 1 Study methods

\begin{tabular}{|c|c|c|c|c|c|}
\hline Paper & Study type & $\begin{array}{l}\text { Follow up } \\
\text { period }\end{array}$ & Number of patients & $\begin{array}{l}\text { Years of } \\
\text { review }\end{array}$ & $\begin{array}{l}\text { GRADE } \\
\text { score }\end{array}$ \\
\hline $\begin{array}{l}\text { Marasco, } 2020, \text { "International } \\
\text { experience using a durable, centrifugal- } \\
\text { flow ventricular assist device for } \\
\text { biventricular support" }\end{array}$ & $\begin{array}{l}\text { Multi-centre retrospective } \\
\text { cohort review }\end{array}$ & 24 months & $\begin{array}{l}93 ; 12 \text { institutions } \\
\text { contributed }\end{array}$ & 2009 to 2017 & ++++ \\
\hline $\begin{array}{l}\text { Maynes, 2020, "Right atrial versus right } \\
\text { ventricular HeartWare HVAD position } \\
\text { in patients on biventricular HeartWare } \\
\text { HVAD support: a systematic review" }\end{array}$ & Systematic Review & $\begin{array}{l}117.5 d \\
(4 \text { months }) \\
(30-342.5)\end{array}$ & $\begin{array}{l}56 \text {; identified } \\
1,288 \text { papers, } \\
\text { included: } 13 \text { papers }\end{array}$ & - & ++++ \\
\hline $\begin{array}{l}\text { Arabia, 2018, "Biventricular support } \\
\text { with intracorporeal, continuous flow, } \\
\text { centrifugal ventricular assist devices" }\end{array}$ & $\begin{array}{l}\text { INTERMACS Registry review- } \\
\text { retrospective review of } \\
\text { prospectively collected data }\end{array}$ & $\begin{array}{l}5.08 \text { months } \\
\text { (157.5 days) }\end{array}$ & $\begin{array}{l}38 ; 19 \text { institutions } \\
\text { contributed }\end{array}$ & $\begin{array}{l}\text { Jun } 2006 \text { to } \\
\text { Jun } 2015\end{array}$ & ++++ \\
\hline $\begin{array}{l}\text { Shah, 2018, "Multicenter experience } \\
\text { with the durable biventricular assist } \\
\text { device" }\end{array}$ & $\begin{array}{l}\text { Multi-centre retrospective } \\
\text { cohort review }\end{array}$ & $\begin{array}{l}6 \text { months } \\
\text { (unreported) }\end{array}$ & $\begin{array}{l}46 ; 6 \text { institutions (of } \\
\text { surgeons }>5 \text { BiVADs) }\end{array}$ & $\begin{array}{l}\text { Oct } 2011 \text { to } \\
\text { Jun } 2017\end{array}$ & ++++ \\
\hline $\begin{array}{l}\text { Lavee, 2018, "An international } \\
\text { multicenter experience of biventricular } \\
\text { support with HeartMate } 3 \text { ventricular } \\
\text { assist systems" }\end{array}$ & $\begin{array}{l}\text { Multi-centre retrospective } \\
\text { cohort review }\end{array}$ & 21 months & $14 ; 6$ centres & $\begin{array}{l}\text { Mar } 2016 \text { to } \\
\text { Jan } 2018\end{array}$ & +++ \\
\hline $\begin{array}{l}\text { Levin, 2016, "Outcomes of } \\
\text { contemporary mechanical circulatory } \\
\text { support device configurations in patients } \\
\text { with severe biventricular failure" }\end{array}$ & $\begin{array}{l}\text { retrospective analysis of UNOS } \\
\text { database }\end{array}$ & $\begin{array}{l}6 \text { months } \\
\text { (unreported) }\end{array}$ & $\begin{array}{l}28 \text { CF-BiVAD } \\
(408 \text { BiVAD/TAH) }\end{array}$ & $\begin{array}{l}\text { Jan } 2010 \text { to } \\
\text { Jun } 2014\end{array}$ & +++ \\
\hline $\begin{array}{l}\text { Maltais, 2016, "Surgical considerations } \\
\text { and challenges for bilateral continuous } \\
\text { flow durable device implantation" }\end{array}$ & Case series & $\begin{array}{l}6 \text { months } \\
\text { (unreported) }\end{array}$ & 4 & $\begin{array}{l}\text { Dec } 2013 \text { to } \\
\text { Aug } 2014\end{array}$ & ++ \\
\hline $\begin{array}{l}\text { Shehab, } 2016, \text { "Long-term biventricular } \\
\text { HeartWare ventricular assist device } \\
\text { support - case series of right atrial and } \\
\text { right ventricular implantation outcomes" }\end{array}$ & Case series & $\begin{array}{l}\text { Census date } \\
\text { Feb } 2015\end{array}$ & 13 & $\begin{array}{l}\text { Aug } 2011 \text { to } \\
\text { Oct } 2014\end{array}$ & +++ \\
\hline
\end{tabular}




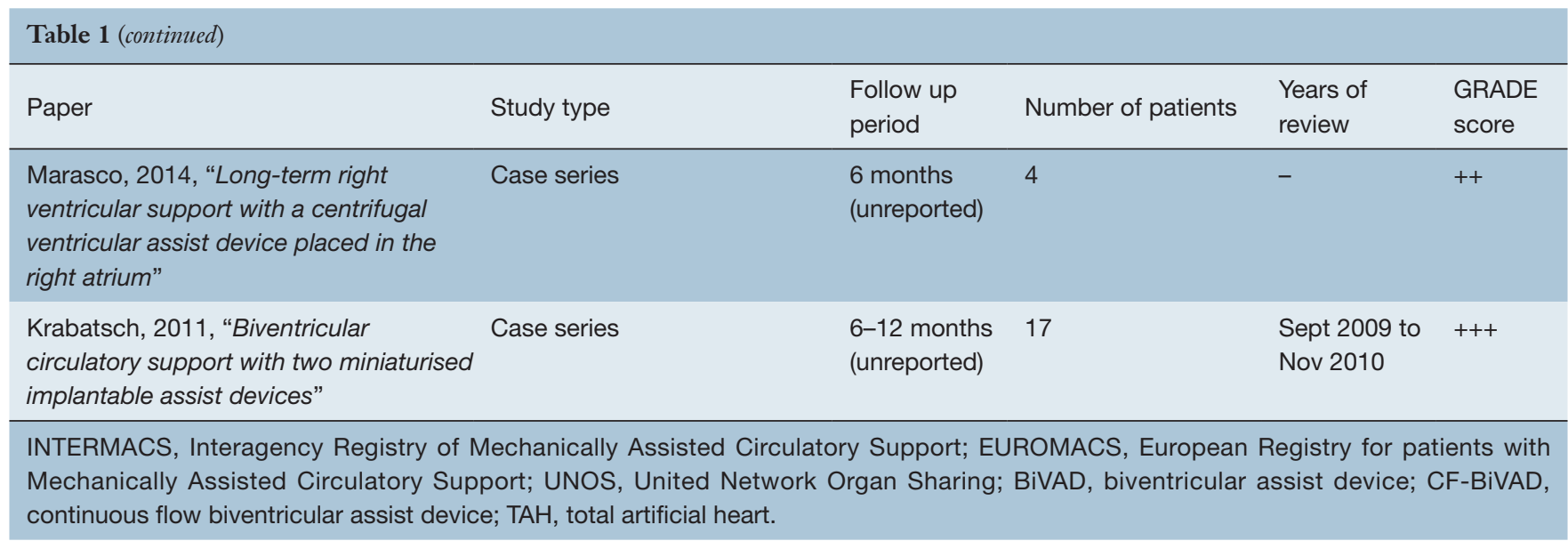

(12 of 14) and was predominantly implanted concomitantly (median $82 \%$, IQR $62-84 \%$ ). Where it was delayed, the median time to implantation was 16.5 days (IQR 10.824.9 days). Cannulation strategy for the RVAD inflow cannula was described in 12 of the 14 studies, and this varied widely with some reporting only RV-cannulation $(25,28)$ whilst others reported an exclusively RA-cannulation approach $(26,29,33)$. In total, 147 had RA-compared with $182 \mathrm{RV}$-inflow cannula cannulation.

\section{Primary outcomes-survival, duration of support, and survival to transplant}

Duration of support was reported in eleven of fourteen papers (Table 4) and was a median of 237 days (IQR 163-309 days). Short-term survival was reported as either 1-month or 30-day survival in ten out of fourteen studies, with a median of $91 \%$ (IQR $82-100 \%$ ). However, only three studies reported survival to discharge, and these were far less successful, ranging from 50-69\%. The 12-month survival was reported in 8 of 14 studies evaluated and ranged from $44-92 \%$, with a median of $59 \%$ (IQR $52.5-65.3 \%$ ). Survival to follow-up was reported in the rest, ranging from 47-91\% (median 69.5\%, IQR 58.8-75\%). Only two studies reported follow-up to 24 months (30,31), reporting 54\% and $56 \%$ survival. Survival to transplant was described in all but one study, and ranged quite significantly, from 3-75\% (median 42\%, IQR 18-50\%).

\section{Secondary outcomes-complications}

ICU length of stay was reported in four studies and had a range of 7-29 days (median 16.5, IQR 12.3-21.5), whilst hospital length of stay (also only reported in the same four studies) ranged from 30 to 53 days (median 41, IQR 36.845.5) (Table 5). Most studies (12 out of 15) reported their pump thrombosis rate, and this varied widely, from 0-75\% (median 30\%, IQR 16-34\%). Pump exchange was lower, with a median $8 \%$ (IQR 3-11\%). Other complications including infection, bleeding, return to theatre, and neurological sequelae were inconsistently reported. These were not comparable across studies, with events per patient year, events per 100 patient months, and percentage affected reported.

\section{Discussion}

Our review demonstrated great variability in study type, follow-up, end-points, device type and configuration making it a challenge to effectively assess the contemporary outcomes of CF-BiVADs.

Study type ranged from registry reviews of INTERMACS, EUROMACS, and UNOS, as well as single- and multi-center cohorts and case series. With likely a great overlap in patient-data recruitment into registries, as well as heterogeneity in end-points, results were thus not compiled to perform a meta-analysis.

Short-term survival was predominantly reported at 30 -day, and as an encouraging median of $91 \%$. However, the survival to discharge reported in only three studies was far less, ranging from $50 \%$ to $69 \%$. This is unsurprising given median hospital length of stay was 41 days. This highlights the protracted and complicated post-operative course of BiVAD recipients, where routine post-operative 
Table 2 Demographics and pre-op status

\begin{tabular}{|c|c|c|c|c|c|c|}
\hline PAPER & Age & Gender & INTERMACS & $\begin{array}{l}\text { Bridge from } \\
\text { ECMO }\end{array}$ & $\begin{array}{l}\text { Primary/secondary } \\
\text { device }\end{array}$ & $\mathrm{CM}$ \\
\hline $\begin{array}{l}\text { Marasco, } 2020 \text {, } \\
\text { "International experience } \\
\text { using a durable, } \\
\text { centrifugal-flow ventricular } \\
\text { assist device for } \\
\text { biventricular support" }\end{array}$ & $\begin{array}{l}\text { Mean: } \\
47.4 \text { years old } \\
\text { (SD 12.9) }\end{array}$ & $\begin{array}{l}70 / 93 \text { male } \\
(75 \%)\end{array}$ & $\begin{array}{l}\text { Int 1-2: } 61 \% \\
\text { Int 1: } 35 \% \\
\text { Int 2: } 26 \% \\
\text { Int 3: } 8 \% \\
\text { Int 4: } 6 \% \\
\text { Unknown: } 25 \%\end{array}$ & $\begin{array}{l}34 \% \\
\text { IABP 5\% }\end{array}$ & - & $\begin{array}{l}\text { Ischaemic } 15 \% \\
\text { Non-ischaemic } 85 \% \\
\text { DCM } 48 \%\end{array}$ \\
\hline $\begin{array}{l}\text { McGiffin, 2020, "The } \\
\text { results of a single- centre } \\
\text { experience with HeartMate } \\
3 \text { in a biventricular } \\
\text { configuration" }\end{array}$ & $\begin{array}{l}\text { Mean: } \\
44 \text { years old } \\
(17 \text { to } 63)\end{array}$ & $\begin{array}{l}12 / 12 \text { male } \\
(100 \%)\end{array}$ & $\begin{array}{l}\text { Int 1-2: } 12 / 12 \\
(100 \%) \\
\text { Int 1: 1/12 (8\%) } \\
\text { Int 2: 11/12 (92\%) }\end{array}$ & $1 / 12(8 \%)$ & All primary & $\begin{array}{l}\text { Non-ischaemic CM } \\
11 / 12(92 \%) \\
6 / 12 \text { DCM }(50 \%) \\
1 / 12 \text { Ischaemic } \\
\text { CM (8\%) }\end{array}$ \\
\hline $\begin{array}{l}\text { Maynes, } 2020 \text {, "Right atrial } \\
\text { versus right ventricular } \\
\text { HeartWare HVAD position } \\
\text { in patients on biventricular } \\
\text { HeartWare HVAD support: } \\
\text { a systematic review" }\end{array}$ & $\begin{array}{l}\text { Median: } \\
51 \text { years old } \\
\text { IQR } 33.8 \text { to } \\
57.0\end{array}$ & $\begin{array}{l}40 / 50 \text { male } \\
(80 \%)\end{array}$ & $\begin{array}{l}\text { Int 1-2: 38/56 } \\
(88.4 \%)\end{array}$ & $9 / 12(75 \%)$ & - & $\begin{array}{l}\text { Non-ischaemic CM: } \\
42 / 56(85.7 \%) \\
\text { Cardiogenic shock: } \\
1 / 56(1.8 \%)\end{array}$ \\
\hline $\begin{array}{l}\text { Vierecke, 2019, "Results } \\
\text { of primary biventricular } \\
\text { support: an analysis of } \\
\text { data from the EUROMACS } \\
\text { registry" }\end{array}$ & $\begin{array}{l}\text { Median: } \\
58 \text { years old } \\
\text { IQR } 39 \text { to } 62\end{array}$ & $\begin{array}{l}20 / 22 \text { male } \\
(91 \%)\end{array}$ & $\begin{array}{l}\text { Int 1: } 2(9 \%) \\
\text { Int 2: } 9(41 \%) \\
\text { Int 3: } 8(36 \%) \\
\text { Int 4: } 3(14 \%)\end{array}$ & $3 / 22(14 \%)$ & All primary & $\begin{array}{l}\text { DCM 8/22 (38\%) } \\
\text { Non-ischaemic } \\
\text { CM: 15/22 (68.2\%) }\end{array}$ \\
\hline $\begin{array}{l}\text { Shah, } 2018, \text { "Multicenter } \\
\text { experience with the } \\
\text { durable biventricular assist } \\
\text { device" }\end{array}$ & $\begin{array}{l}\text { Median: } \\
46 \text { years old } \\
\text { IQR } 19 \text { to } 67\end{array}$ & $\begin{array}{l}36 / 46 \text { male } \\
(78 \%)\end{array}$ & $\begin{array}{l}\text { Int 1: 32/46 (70\%) } \\
\text { Int 2: 10/46 (22\%) } \\
\text { Int 3: } 4 / 46(8 \%)\end{array}$ & $\begin{array}{l}\text { 12/46 (26\%) } \\
\text { IABP 44\% }\end{array}$ & All primary & $\begin{array}{l}\text { Non-ischaemic CM: } \\
37 / 46(80 \%) \\
\text { Ischaemic CM: } 6 / 46 \\
(13 \%)\end{array}$ \\
\hline $\begin{array}{l}\text { Lavee, } 2018, \text { "An } \\
\text { international multicenter } \\
\text { experience of biventricular } \\
\text { support with HeartMate } 3 \\
\text { ventricular assist systems" }\end{array}$ & $\begin{array}{l}\text { Median: } \\
48.5 \text { years old } \\
\text { (17 to } 73 \text { ) }\end{array}$ & $\begin{array}{l}13 / 14 \text { male } \\
(93 \%)\end{array}$ & $\begin{array}{l}\text { Int 1: } 2 / 14(14 \%) \\
\text { Int 2: } 9 / 14(64 \%) \\
\text { Int 3: } 3 / 14(21 \%)\end{array}$ & - & - & $\begin{array}{l}\text { Non-ischaemic CM: } \\
\text { 10/14 (71\%) } \\
\text { DCM: } 7 / 14(50 \%) \\
\text { Ischaemic CM: } 4 / 14 \\
(29 \%)\end{array}$ \\
\hline
\end{tabular}

Table 2 (continued) 
Table 2 (continued)

\begin{tabular}{|c|c|c|c|c|c|c|}
\hline PAPER & Age & Gender & INTERMACS & $\begin{array}{l}\text { Bridge from } \\
\text { ECMO }\end{array}$ & $\begin{array}{l}\text { Primary/secondary } \\
\text { device }\end{array}$ & $\mathrm{CM}$ \\
\hline $\begin{array}{l}\text { Eulert-Grehn, 2018, "Two } \\
\text { implantable continuous- } \\
\text { flow ventricular assist } \\
\text { devices in a biventricular } \\
\text { configuration: technique } \\
\text { and results" }\end{array}$ & $\begin{array}{l}\text { Mean: } \\
52 \text { years old } \\
(21 \text { to } 73)\end{array}$ & - & - & - & - & $\begin{array}{l}\text { DCM: } 17 / 39(44 \%) \\
\text { Ischaemic CM: } 13 \\
(33 \%)\end{array}$ \\
\hline $\begin{array}{l}\text { Tran, 2018, "Durable } \\
\text { biventricular support using } \\
\text { right atrial placement of } \\
\text { the HeartWare HVAD" }\end{array}$ & $\begin{array}{l}\text { Mean: } \\
42.3 \text { years old } \\
(19 \text { to } 57)\end{array}$ & $\begin{array}{l}9 / 11 \text { male } \\
(82 \%)\end{array}$ & $\begin{array}{l}\text { Int 1/2: } 11 / 11 \\
(100 \%) \\
\text { Int 1: } 7 / 11(64 \%) \\
\text { Int 2: } 4 / 11(36 \%)\end{array}$ & - & - & $\begin{array}{l}\text { Ischaemic CM: } 1 / 11 \\
(9 \%) \\
\text { Non-ischaemic CM: } \\
10 / 11(91 \%)\end{array}$ \\
\hline $\begin{array}{l}\text { Maltais, 2016, "Surgical } \\
\text { considerations and } \\
\text { challenges for bilateral } \\
\text { continuous flow durable } \\
\text { device implantation" }\end{array}$ & $\begin{array}{l}\text { Mean: } \\
34 \text { years old } \\
(18 \text { to } 63 \text { ) }\end{array}$ & - & $\begin{array}{l}\text { Int } 1: 2 / 4 \\
\text { Int 2: } 1 / 4 \\
\text { Int 3: } 1 / 4\end{array}$ & $1 / 4$ & - & $\begin{array}{l}\text { Viral } 1 / 4 \\
\text { Post-partum } 1 / 4 \\
\text { Ischaemic } 1 / 4 \\
\text { Familial } 1 / 4\end{array}$ \\
\hline $\begin{array}{l}\text { Shehab, 2016, "Long-term } \\
\text { biventricular HeartWare } \\
\text { ventricular assist device } \\
\text { support - case series } \\
\text { of right atrial and right } \\
\text { ventricular implantation } \\
\text { outcomes" }\end{array}$ & $\begin{array}{l}\text { Mean: } \\
45 \text { years old } \\
(S D 11)\end{array}$ & $\begin{array}{l}\text { 10/13 male } \\
(77 \%)\end{array}$ & $\begin{array}{l}\text { Int 1: 10/13 (77\%) } \\
\text { Int 2: } 3 / 13(23 \%)\end{array}$ & $\begin{array}{l}\text { ECMO 5/13 } \\
(38 \%) \\
\text { IABP 2/13 } \\
(15 \%)\end{array}$ & - & DCM: 11/13 (85\%) \\
\hline $\begin{array}{l}\text { Krabatsch, 2011, } \\
\text { "Biventricular circulatory } \\
\text { support with two } \\
\text { miniaturised implantable } \\
\text { assist devices" }\end{array}$ & $\begin{array}{l}\text { Mean: } 51.8 \\
\text { years old ( } 29 \\
\text { to } 73)\end{array}$ & $\begin{array}{l}15 / 17 \text { male } \\
(88 \%)\end{array}$ & $\begin{array}{l}\text { Int 1/2: } 11 / 17 \\
(65 \%) \\
\text { Int 3/4: 5/17 } \\
(30 \%)\end{array}$ & - & - & $\begin{array}{l}\text { DCM: } 9 / 17 \text { (53\%) } \\
\text { Ischaemic CM: 4/17 } \\
(24 \%)\end{array}$ \\
\hline
\end{tabular}




\begin{tabular}{|l|l|l|}
\hline Profile & Title & Description \\
\hline 1 & Critical cardiogenic shock & Life-threatening hypotension refractory to IV inotropes. "crash and burn" \\
\hline 2 & Progressive decline & IV inotropes required with worsening end-organ function. "sliding on inotropes" \\
\hline 3 & Inotrope dependent & Stable blood pressure and end-organ function but failure to wean from IV inotropes. "dependent stability" \\
\hline 4 & Resting symptoms & Daily symptoms of congestion at rest or with ADLs. High doses of diuretics \\
\hline 5 & Exertion intolerant & Unable to engage in any activity above ADLs \\
\hline 6 & Exertion limited & Can participate in minor activities but quickly fatigues. "walking wounded" \\
\hline 7 & Advanced NYHA III & Comfortable with meaningful activity, limited to mild exertion \\
\hline
\end{tabular}

Figure 1 INTERMACS profiles. INTERMACS, Interagency Registry for Mechanically Assisted Circulatory Support; NYHA, New York Heart Association; IV, intra-venous; ADLs, activities of daily living.

Table 3 Devices, configurations and technique

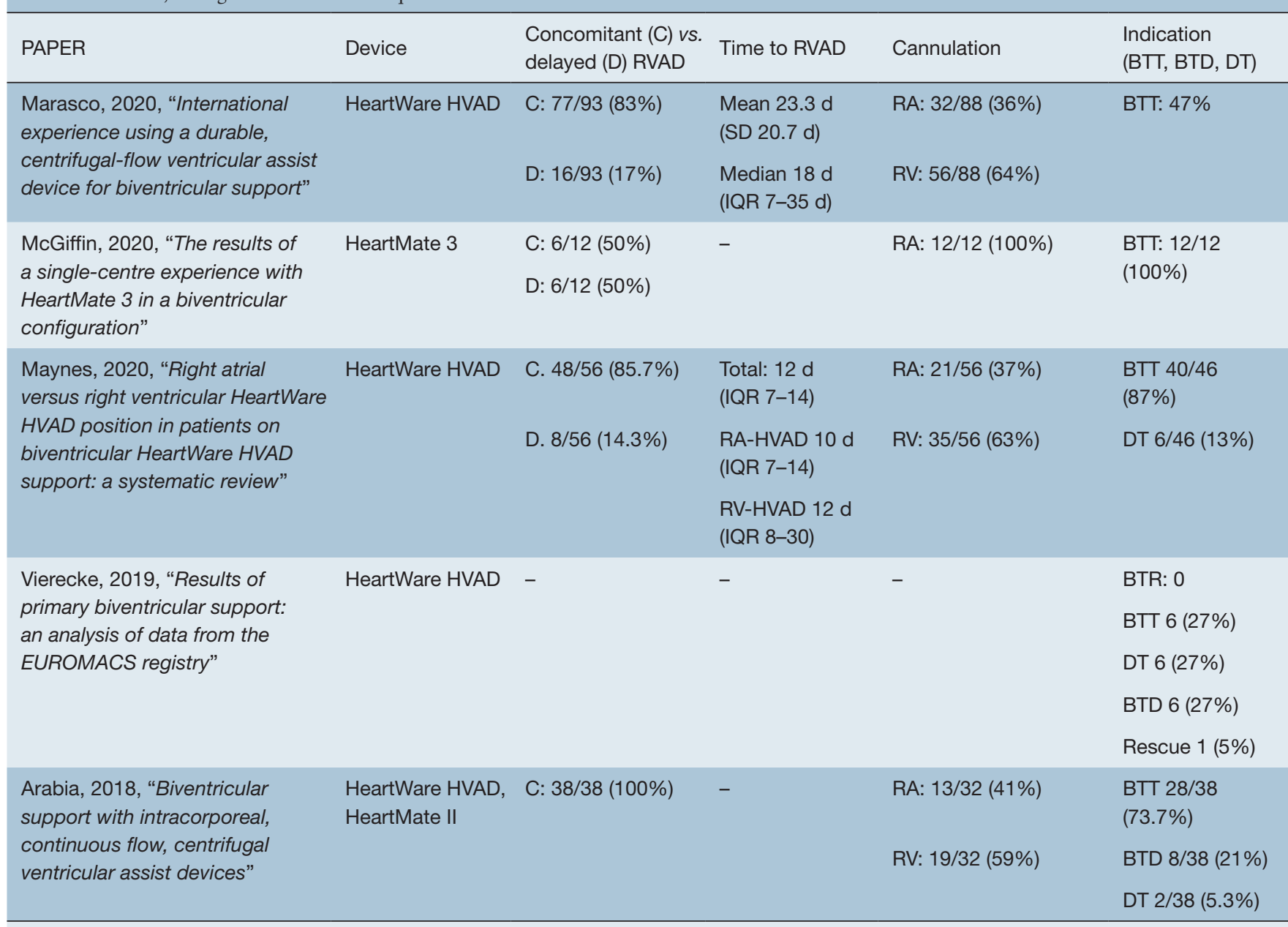

Table 3 (continued) 
Table 3 (continued)

\begin{tabular}{|c|c|c|c|c|c|}
\hline PAPER & Device & $\begin{array}{l}\text { Concomitant (C) vs. } \\
\text { delayed (D) RVAD }\end{array}$ & Time to RVAD & Cannulation & $\begin{array}{l}\text { Indication } \\
\text { (BTT, BTD, DT) }\end{array}$ \\
\hline \multirow{2}{*}{$\begin{array}{l}\text { Shah, 2018, "Multicenter } \\
\text { experience with the durable } \\
\text { biventricular assist device" }\end{array}$} & \multirow[t]{2}{*}{ HeartWare HVAD } & C: $31 / 46(67 \%)$ & \multirow[t]{2}{*}{$15 \mathrm{~d}(13$ to 30$)$} & RA: $23 / 46(50 \%)$ & $\begin{array}{l}\text { ВТT 38/46 } \\
(83 \%)\end{array}$ \\
\hline & & D: 15/46 (33\%) & & RV: 23/46 (50\%) & DT 8/46 (17\%) \\
\hline \multirow{2}{*}{$\begin{array}{l}\text { Lavee, 2018, "An international } \\
\text { multicenter experience of } \\
\text { biventricular support with } \\
\text { HeartMate } 3 \text { ventricular assist } \\
\text { systems" }\end{array}$} & \multirow[t]{2}{*}{ HeartMate 3} & C: $8 / 14(57 \%)$ & \multirow[t]{2}{*}{$45.6 \mathrm{~d}$ (9 to 112$)$} & RA: 12/14 (86\%) & \multirow[t]{2}{*}{ - } \\
\hline & & & & $\begin{array}{l}\text { Ventricular excision and } \\
\text { TAH Config: 1/14 (7\%) }\end{array}$ & \\
\hline \multirow{2}{*}{$\begin{array}{l}\text { Eulert-Grehn, 2018, "Two } \\
\text { implantable continuous-flow } \\
\text { ventricular assist devices in } \\
\text { a biventricular configuration: } \\
\text { technique and results" }\end{array}$} & \multirow{2}{*}{$\begin{array}{l}\text { HeartWare HVAD } \\
\text { HeartMate } 3\end{array}$} & C: $22 / 39$ (56\%) & \multirow[t]{2}{*}{-} & RA: $17 / 36(47 \%)$ & \multirow[t]{3}{*}{ - } \\
\hline & & D: $17 / 39$ (44\%) & & RV: 19/36 (53\%) & \\
\hline $\begin{array}{l}\text { Tran, 2018, "Durable biventricular } \\
\text { support using right atrial } \\
\text { placement of the HeartWare } \\
\text { HVAD" }\end{array}$ & $\begin{array}{l}\text { RVAD: HeartWare } \\
\text { HVAD } 11 / 11\end{array}$ & D: $2 / 11(18 \%)$ & D: Within 1 week & RA: $11 / 11$ & \\
\hline $\begin{array}{l}\text { Levin, 2016, "Outcomes of } \\
\text { contemporary mechanical } \\
\text { circulatory support device } \\
\text { configurations in patients with } \\
\text { severe biventricular failure" }\end{array}$ & - & - & - & - & BTT $100 \%$ \\
\hline \multirow[t]{2}{*}{$\begin{array}{l}\text { Marasco, 2014, "Long-term } \\
\text { right ventricular support with } \\
\text { a centrifugal ventricular assist } \\
\text { device placed in the right atrium" }\end{array}$} & $\begin{array}{l}\text { LVAD: } 3 / 4 \\
\text { HeartWare HVAD; } \\
\text { 1/4 VentrAssist }\end{array}$ & C: $1 / 4$ D: $3 / 4$ & \multirow[t]{2}{*}{$\begin{array}{l}\mathrm{D}: 3 / 4-18 \mathrm{~d} \\
\text { mean (7 to 33) }\end{array}$} & $\mathrm{RA}: 4 / 4$ & \multirow[t]{2}{*}{ ВТТ: $4 / 4$} \\
\hline & $\begin{array}{l}\text { RVAD: } 4 / 4 \\
\text { HeartWare HVAD }\end{array}$ & & & & \\
\hline \multirow{2}{*}{$\begin{array}{l}\text { Krabatsch, 2011, "Biventricular } \\
\text { circulatory support with two } \\
\text { miniaturised implantable assist } \\
\text { devices" }\end{array}$} & \multirow[t]{2}{*}{ HeartWare HVAD } & C: 14/17 (82\%) & \multirow[t]{2}{*}{-} & RV: 17/17 (100\%) & \multirow{2}{*}{$\begin{array}{l}\text { BTT: } 13 / 17 \\
\text { (76\%) DT: } 4 / 17 \\
(24 \%)\end{array}$} \\
\hline & & D: $3 / 17(18 \%)$ & & $\begin{array}{l}\text { RA: } 1 / 17 \text { (switched } \\
\text { from RV) }\end{array}$ & \\
\hline
\end{tabular}

RVAD, right ventricular assist device; LVAD, left ventricular assist device; BiVAD, biventricular assist device; RA, right atrial; RV, right ventricular; BTT, bridge to transplant; BTD, bridge to decision; DT, destination therapy. 
HeartWare HVAD

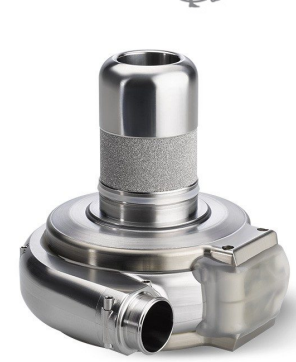

Marasco, 2020

Maynes, 2020

McGiffin, 2020

Eulert-Grehn, 2018

Lavee, 2018

Vierecke, 2019

Arabia, 2018 *

Shah, 2018

Tran, 2018 *

Shehab, 2016

Maltais, 2016

Marasco, 2014 *

Krabatsch, 2011

Levin, 2016 **

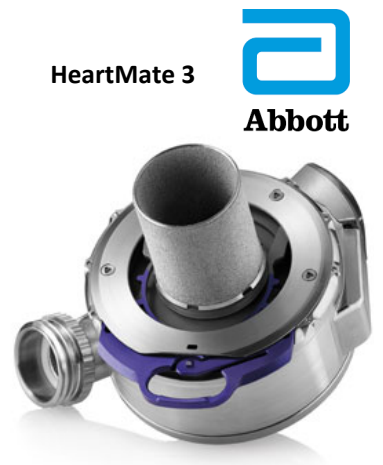

* Utilised other device in LVAD (HeartMate 2 and Ventrassist), but HVAD for RVAD

** Did not specify which devices used

Figure 2 Devices evaluated by studies.

Table 4 Results-primary outcomes

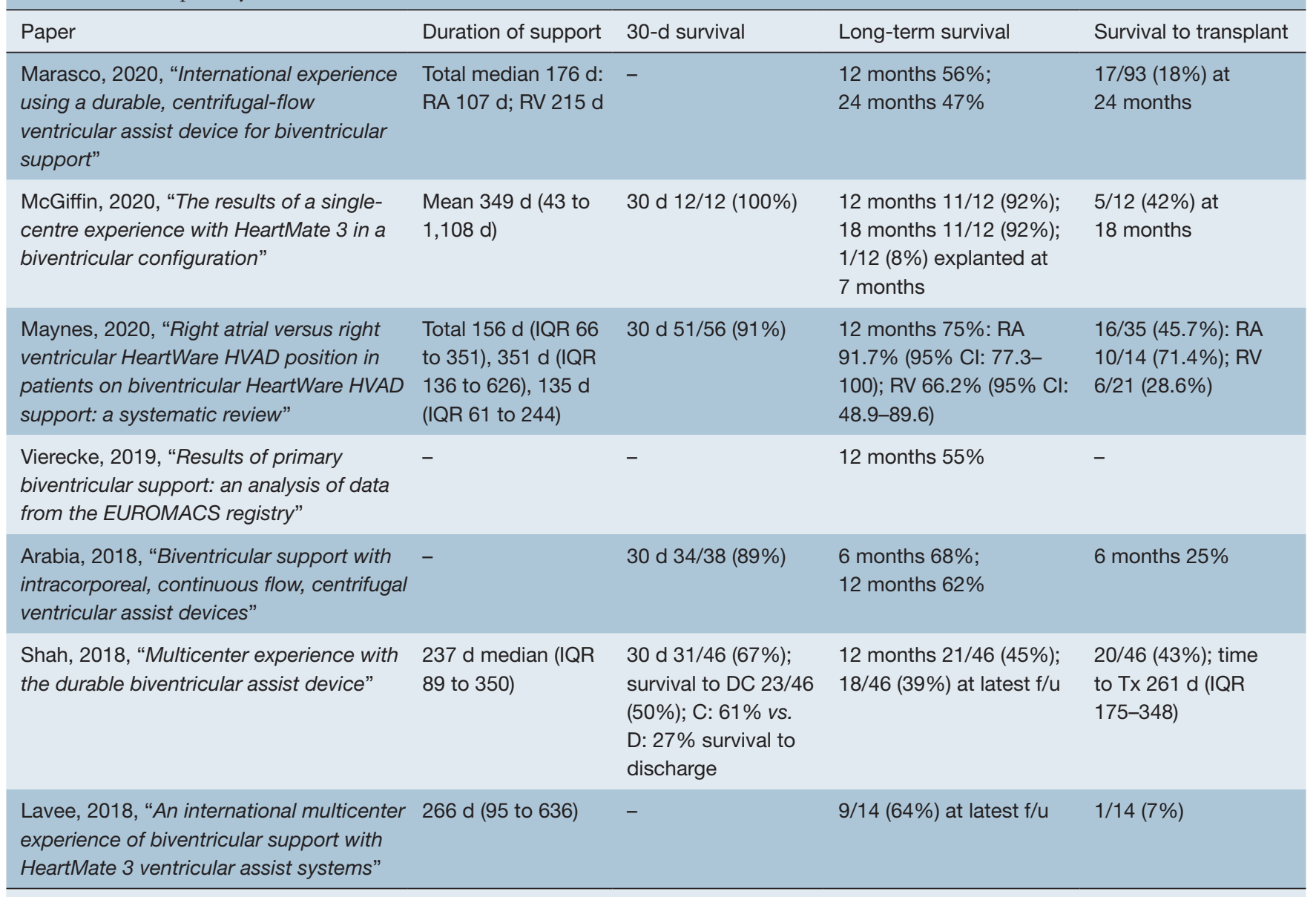

Table 4 (continued) 
Table 4 (continued)

\begin{tabular}{|c|c|c|c|c|}
\hline Paper & Duration of support & 30-d survival & Long-term survival & Survival to transplant \\
\hline $\begin{array}{l}\text { Levin, 2016, "Outcomes of contemporary } \\
\text { mechanical circulatory support device } \\
\text { configurations in patients with severe } \\
\text { biventricular failure" }\end{array}$ & $97.5 \mathrm{~d}$ (52.5 to 151$)$ & $\begin{array}{l}1 \text { month survival } \\
82 \%\end{array}$ & 6 months survival $57 \%$ & $\begin{array}{l}\text { Tx } 6 \text { months post- } \\
\text { BiVAD } 21 / 28(75 \%) \\
83 \% 6 \text { months } \\
\text { survival post- } \\
\text { transplant }\end{array}$ \\
\hline $\begin{array}{l}\text { Shehab, 2016, "Long-term biventricular } \\
\text { HeartWare ventricular assist device } \\
\text { support - case series of right atrial and } \\
\text { right ventricular implantation outcomes" }\end{array}$ & $\begin{array}{l}\text { Median } 269 \text { d (IQR } \\
93 \text { to } 426)\end{array}$ & $\begin{array}{l}30 \text { d survival } 13 / 13 \\
(100 \%) ; \text { survival to } \\
\text { discharge } 9 / 13(69 \%)\end{array}$ & $\begin{array}{l}12 \text { months } 8 / 13(62 \%) \text {, } \\
24 \text { months } 7 / 13(54 \%)\end{array}$ & $\begin{array}{l}5 / 11(38 \%) \text { at median } \\
513 \text { d support }\end{array}$ \\
\hline $\begin{array}{l}\text { Marasco, 2014, "Long-term right } \\
\text { ventricular support with a centrifugal } \\
\text { ventricular assist device placed in the right } \\
\text { atrium" }\end{array}$ & $\begin{array}{l}\text { Mean } 503 d(117 \text { to } \\
772)\end{array}$ & 1 month survival $4 / 4$ & $3 / 4$ at latest $f / u$ & $\begin{array}{l}2 / 4(50 \%) 1 / 4 \\
\text { pending } T x\end{array}$ \\
\hline $\begin{array}{l}\text { Krabatsch, 2011, "Biventricular circulatory } \\
\text { support with two miniaturised implantable } \\
\text { assist devices" }\end{array}$ & $\begin{array}{l}\text { Mean } 170 \mathrm{~d}(\mathrm{SD} \\
163)\end{array}$ & $\begin{array}{l}30 \text { d survival } 14 / 17 \\
\text { (82\%); survival to } \\
\text { discharge } 10 / 17 \\
(59 \%)\end{array}$ & $8 / 17(47 \%)$ at latest $f / u$ & $\begin{array}{l}1 / 17(6 \%) \\
\text { transplanted }(280 \mathrm{~d})\end{array}$ \\
\hline
\end{tabular}

markers of success are less relevant and accurate.

Long-term survival was less consistently reported, ranging from 6 to 24 months, and survival to follow-upwhere a time-interval was often not specified. Duration of follow-up was seldom longer than 12 months which reflects the relatively diminutive number of BiVAD recipients, the largely bridge to transplant cohort, as well as the novel nature of continuous-flow devices in this configuration. Nonetheless, survival when reported at 12 months was $59 \%$, significantly lower than at 30-day, and highlights how critically unwell this cohort is. BiVAD implantation confers double the risk of mortality at 12 months compared with LVAD alone (20,34), however, no singular factor has been identified as to why BiVADs are associated with higher mortality. Several studies have shown that certain indices of RVF including right atrial pressure (RAP) and serum bilirubin secondary to hepatic congestion are independent risk factors. Therefore, it may be the chronicity of RVF and the associated end-organ dysfunction (or sequalae of delayed utilisation in acute RHF) — rather than the device and its potential complications-which contribute the greatest to post-operative mortality (35).

Demographics across cohorts were relatively similar, and this was most demonstrable with the etiology of cardiomyopathy and INTERMACS profile. Across all studies, most candidates suffered a non-ischaemic cardiomyopathy. Severity of heart-failure was most consistently measured with the INTERMACS profile, and BiVAD recipients were predominantly INTERMACS 1-2. Once again, this impacts survival; BiVAD compared with 


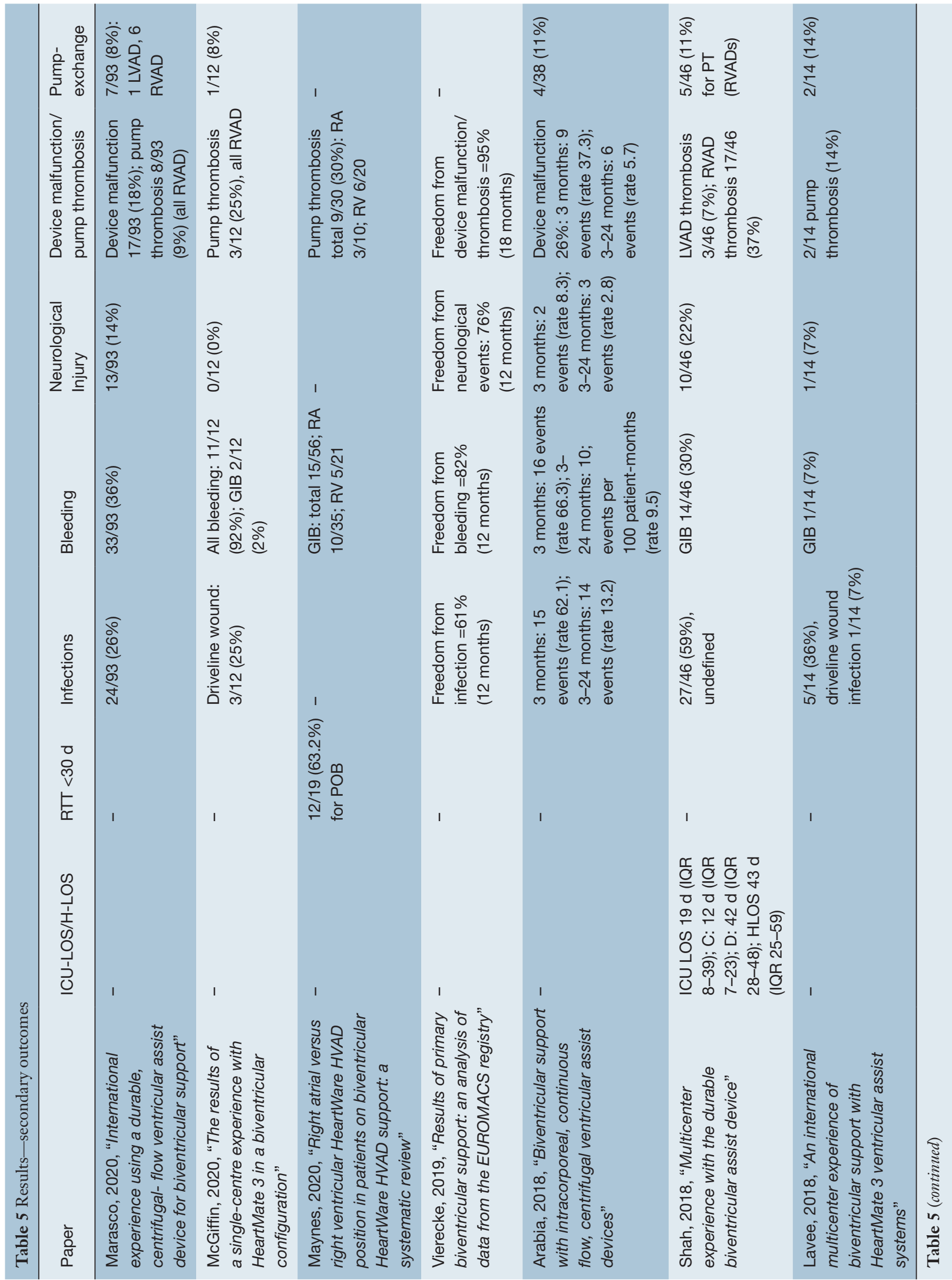




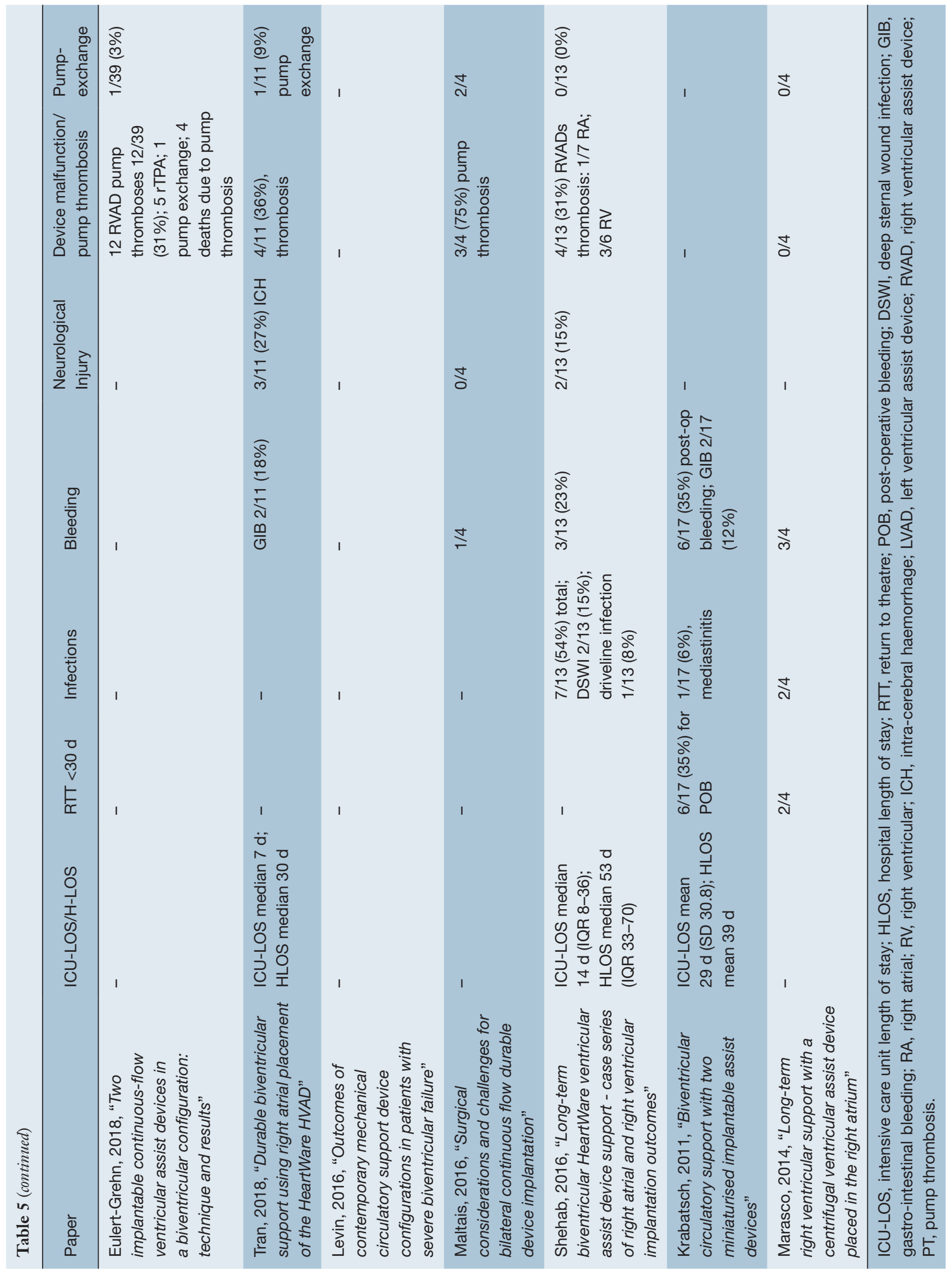




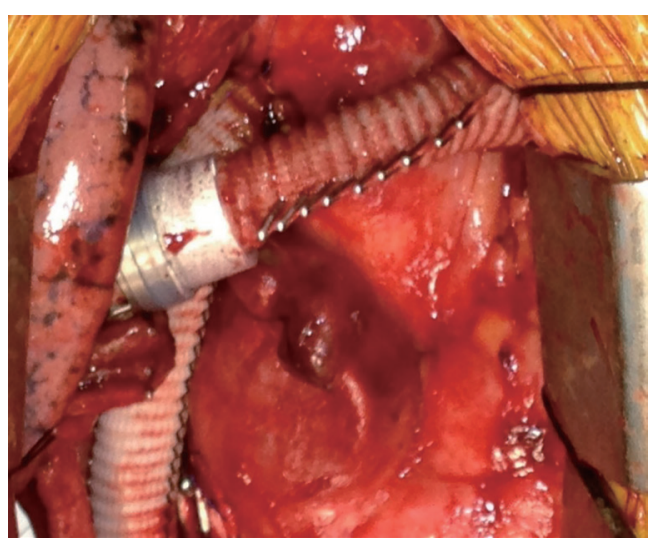

Figure 3 RVAD outflow graft (crossing the ascending aorta) crimped with hemaclips (reprinted with authors permission) (23).

LVAD recipients are by far more critically unwell and in cardiogenic shock-likely contributing to poorer long-term survival and survival to transplant. Although transplant would be the ideal management, their critical pre-operative condition and multi-organ dysfunction likely reduces their candidacy (24).

The indication of LVADs is significantly different to BiVAD implantation. The proportion of LVADs implanted for destination therapy has progressively increased from $46 \%$ in 2014 to $70 \%$ in 2019 (21). Most patients in this review were implanted with BiVADs with the intent of bridge to transplantation. However, there is discrepancy across global regions where locality impacts overall patient selection leading to heterogenous indications for VAD implantation across the international community. Whereas VADs are indicated as bridge to transplant, destination therapy and even bridge to candidacy (decision) in the USA and Europe, LVADs are not approved for destination therapy in Japan (36). The relevance of indication is heightened by the fact that wait-list times for heart transplantation vary widely with Japan having a median of more than 1,150 days (37). This is significantly higher than the global wait-list median of 144 days reported in 2014 (38). Thus, this is likely to affect candidacy for BiVAD support and patient selection across regions.

Continuous-flow devices hold several advantages over their pulsatile predecessors that are unique to the BiVAD setting. Pulsatile biventricular devices were initially utilized but were limited by their large size, requiring extensive pockets to be created in the abdominal wall, and with four cannulae exiting/entering the skin for externalized pumps, increasing risk of infection (28). Additionally, their drivers were bulkier and pumps more prone to thrombosis (17).

In our systematic review, complications were too inconsistently reported to be comparable. Of greatest interest is the incidence of pump thrombosis and need for pump-exchange. The median incidence of pump thrombosis was $30 \%$ across the larger cohorts studied with a subsequent $8 \%$ incidence of pump exchange. Although most pump thromboses reported were thrombolyzed to avert pump exchange, almost all thrombosis complications were of the RVAD. Pump thrombosis and exchange remains the main dilemma with BiVADs, whether continuous-flow or otherwise.

As it is primarily designed for the left ventricle, these LVADs implanted do not take into account the anatomy, geometry and physiology of the right ventricle. As such, the inlet is too long (primarily designed for LV apex cannulation) and pump flows too high [not accounting for the lower afterload of pulmonary vascular resistance (PVR) compared with systemic (SVR)]. The HeartWare HVAD for example accommodates for $600-3,500$ dynes $/ \mathrm{sec} / \mathrm{cm}^{5}$. However, PVR is generally $<250$ dynes, and banding procedures on the VAD outflow trunk are usually required to increase the afterload, and thus reduce the risk of pulmonary oedema from excessive flow to the pulmonary vasculature (28). Across our review, each study described a differing surgical approach, from: inlet cannulation, pumpplacement, pump-speed variation, banding strategy, and even TV explantation. Within some studies, there were sub-group analyses comparing RA- and RV-cannulation.

Reducing outflow graft diameter with suture line, clips (Figure 3), bands, or utilizing an $8 \mathrm{~mm}$ outflow graft rather than the standard $10 \mathrm{~mm}$ diameter have all been described in both the HeartWare HVAD and HeartMate 3 (26,39). This increases resistance to the pump so that a lower PVR can be accommodated. Modifications of pump speed to accommodate for reduced PVR have been evaluated, but must be balanced against the increased risk of pump thrombosis at lower speeds. These are obviously modelspecific, and thus modifications to one device are not uniformly applicable across other devices (40).

All studies describing their configuration detailed procedures to reduce the inflow-cannula intra-cavity distance, predominantly with felt-spacers. Furthermore, our review found that $45 \%$ of BiVAD recipients had RA-inflow cannulation compared with $55 \% \mathrm{RV}$-inflow cannulation of the RVAD. Higher rate of thrombosis has been most often found in RV-inflow cannulation (24), with the advent of RAinflow cannulation designed to combat this complication. 
With the offloading of both ventricles, the RV has been visualized to remodel on serial echocardiography, resulting in a reduced cavity size. As such, suction events become more frequent leading to a higher incidence of pump thrombosis. This has been dynamically demonstrated in multiple patients with occlusion of the inflow cannula by the interventricular septum on respiratory expiration (30).

TV-explantation was performed by a small case series of four patients by Maltais et al., with the intent of reducing RVAD thrombosis with RV-inflow cannulation (25). However, not only does this preclude the possibility of RVAD removal in RV recovery, three out of four of their patients had pump-thrombosis nonetheless.

Delayed RVAD implantation occurred in $18 \%$ of cases across studies. Often this was preceded with a temporary mechanical support device to trial need for RVAD, as it was presumably an unexpected event. Nonetheless, delayed RVAD implantation is proven to have worse outcomes than planned (concomitant) RVAD implantation, although we could not accurately compare long-term outcomes in our study $(4,24,41)$.

But there remain limitations to BiVADs that undoubtedly reduce survival and quality of life compared with LVAD alone, prompting many clinicians to avoid RVAD implantation with temporary devices if possible. There remain two separate drivelines, and if two different devices are implanted (occasionally in delayed RVAD implantation) having two separate controllers is of great inconvenience for the patient and care-team. Although risk of infection is increased with two drivelines, size is also of great significance. Solutions to extracorporeal power supply have been attempted. The Lion Heart study examining a completely implantable device without a driveline and requiring only transcutaneous power induction was associated with significant infection risk. This was thought to be secondary to volume and size of foreign material located within the chest (42). As devices become smaller, risk of infection is likely to decrease as well.

Some institutions are reticent to implement BiVAD support to transplant due to potentially worse outcomes than single device, increased cost, demanding and nonstandardized implantation techniques, and reduced patient quality of life with double peripherals (43). However, in centers where transplant wait-list times are shorter, there may be less reluctance to place high-risk patients on BiVAD and thus ameliorate the risk of delayed insertion (43). In fact, BiVAD implantation has been recommended to be limited to BTT patients rather than destination therapy
(DT), where non-survival outcomes are preferenced including quality of life and functionality (13). As such, higher risk profile groups (INTERMACS 1-2) are less likely to receive VAD support if BTT is unlikely, and LVAD for DT is becoming a more semi-elective procedure (44).

There remains the TAH as a viable alternative to BiVAD implantation. Studies show no difference in outcome between TAH vs. BiVAD thus far $(20,45)$, and the single driveline is seemingly an attractive alternative. However, TAH requires explantation of the native ventricles, and thus excludes the potential for ventricular recovery, which was demonstrated to be $5 \%$ by Cleveland et al. 2011 (14). It is also bulkier, more expensive, and few centers have experience with its implantation and post-operative care, compared with utilizing an additional LVAD (44). Furthermore, being a pulsatile device, it requires a large driver that contains a noisy compressor and is significantly limiting in comparison with the BiVADs currently available (28). However, TAH does provide a role in patients with refractory arrhythmias, as well as those with restrictive cardiomyopathies where VAD placement would be complicated by small ventricular cavities.

Overall, the need for a biventricular assist device presents a conundrum. There is no dedicated long-term RV support device and in such severe biventricular failure, transplant would be the best option. The staged approach of RVAD implantation demonstrates the unpredictable nature of RVF post LVAD insertion. And, although most VAD recipients are indicated for destination therapy, the opposite trend is found with BiVADs recipients, whom are predominantly indicated as bridge to transplant.

\section{Limitations}

There was significant overlap in the cohorts studied, with many studies including populations from one another $(23,27,28)$. In particular, there would be overlap of patient cohorts from various registries including UNOS, InterMACS, and EuroMACS, which likely introduces bias to the results. Unfortunately, these cohorts could not be separated to reveal the absolute number of patients. Additionally, small sample size bias may have affected study results-the largest being $93 \mathrm{CF}-\mathrm{BiVAD}$ recipients. Once again this demonstrates the novel nature of continuous-flow devices being utilized in this niche heart failure strategy. With no particular VAD designed for the right ventricle, the differing strategies of conforming an LVAD for RV use adds heterogeneity. This may have confounded the results 
further with differing anticoagulation strategies postoperatively also described. Finally, there was no consistent indication algorithm for BiVAD implantation across studies-some studies having defined indications based upon RV failure parameters (40), whilst others deferred to surgeons' discretion (27).

\section{Conclusions}

Although these patients represent a small subset of cardiac failure patients requiring mechanical ventricular assistance, a dedicated CF-RVAD for a BiVAD configuration is greatly in need. The variety of alterations and adjustments to off-label use of varying LVADs demonstrates how unregulated practice can be. A purpose-designed device would greatly reduce error by standardizing practice whilst reducing risk of pump thrombosis. A dedicated BiVAD device would ideally taper to a single driveline, or better, a trans-cutaneous charge thus reducing infection risk and improving quality of life. Studies are promising in the advent of continuous-flow devices, and greater progress is now needed to accommodate for this cohort whilst awaiting definitive transplantation.

\section{Acknowledgments}

Funding: None.

\section{Footnote}

Conflicts of Interest: The authors have no conflicts of interest to declare.

Open Access Statement: This is an Open Access article distributed in accordance with the Creative Commons Attribution-NonCommercial-NoDerivs 4.0 International License (CC BY-NC-ND 4.0), which permits the noncommercial replication and distribution of the article with the strict proviso that no changes or edits are made and the original work is properly cited (including links to both the formal publication through the relevant DOI and the license). See: https://creativecommons.org/licenses/by-nc-nd/4.0/.

\section{References}

1. Kormos RL, Teuteberg JJ, Pagani FD, et al. Right ventricular failure in patients with the HeartMate II continuous-flow left ventricular assist device: incidence, risk factors, and effect on outcomes. J Thorac Cardiovasc Surg 2010;139:1316-24.

2. Takeda K, Naka Y, Yang JA, et al. Timing of temporary right ventricular assist device insertion for severe right heart failure after left ventricular assist device implantation. ASAIO J 2013;59:564-9.

3. Eisen HJ. Left Ventricular Assist Devices (LVADS): History, Clinical Application and Complications. Korean Circ J 2019;49:568-85.

4. Takeda K, Naka Y, Yang JA, et al. Outcome of unplanned right ventricular assist device support for severe right heart failure after implantable left ventricular assist device insertion. J Heart Lung Transplant 2014;33:141-8.

5. Dang NC, Topkara VK, Mercando M, et al. Right heart failure after left ventricular assist device implantation in patients with chronic congestive heart failure. J Heart Lung Transplant 2006;25:1-6.

6. Fitzpatrick JR 3rd, Frederick JR, Hsu VM, et al. Risk score derived from pre-operative data analysis predicts the need for biventricular mechanical circulatory support. J Heart Lung Transplant 2008;27:1286-92.

7. Matthews JC, Koelling TM, Pagani FD, et al. The right ventricular failure risk score a pre-operative tool for assessing the risk of right ventricular failure in left ventricular assist device candidates. J Am Coll Cardiol 2008;51:2163-72.

8. Atluri P, Goldstone AB, Fairman AS, et al. Predicting right ventricular failure in the modern, continuous flow left ventricular assist device era. Ann Thorac Surg 2013;96:857-63; discussion 863-4.

9. Potapov EV, Stepanenko A, Dandel M, et al. Tricuspid incompetence and geometry of the right ventricle as predictors of right ventricular function after implantation of a left ventricular assist device. J Heart Lung Transplant 2008;27:1275-81.

10. Kukucka M, Stepanenko A, Potapov E, et al. Right-toleft ventricular end-diastolic diameter ratio and prediction of right ventricular failure with continuous-flow left ventricular assist devices. J Heart Lung Transplant 2011;30:64-9.

11. Pettinari M, Jacobs S, Rega F, et al. Are right ventricular risk scores useful? Eur J Cardiothorac Surg 2012;42:621-6.

12. Potapov EV, Schoenrath F, Falk V. Clinical signs of right ventricular failure following implantation of a left ventricular assist device. Eur J Heart Fail 2020;22:383-4.

13. Kiernan MS, Grandin EW, Brinkley M Jr, et al. Early Right Ventricular Assist Device Use in Patients Undergoing Continuous-Flow Left Ventricular Assist 
Device Implantation: Incidence and Risk Factors From the Interagency Registry for Mechanically Assisted Circulatory Support. Circ Heart Fail 2017;10:e003863.

14. Cleveland JC Jr, Naftel DC, Reece TB, et al. Survival after biventricular assist device implantation: an analysis of the Interagency Registry for Mechanically Assisted Circulatory Support database. J Heart Lung Transplant 2011;30:862-9.

15. Arabía FA, Milano CA, Mahr C, et al. Biventricular Support With Intracorporeal, Continuous Flow, Centrifugal Ventricular Assist Devices. Ann Thorac Surg 2018;105:548-55.

16. Maynes EJ, O'Malley TJ, Patel P, et al. Right atrial versus right ventricular HeartWare HVAD position in patients on biventricular HeartWare HVAD support: A systematic review. Artif Organs 2020;44:926-34.

17. Slaughter MS, Rogers JG, Milano CA, et al. Advanced heart failure treated with continuous-flow left ventricular assist device. N Engl J Med 2009;361:2241-51.

18. Pagani FD, Miller LW, Russell SD, et al. Extended mechanical circulatory support with a continuous-flow rotary left ventricular assist device. J Am Coll Cardiol 2009;54:312-21.

19. Frazier OH, Myers TJ, Gregoric I. Biventricular assistance with the Jarvik FlowMaker: a case report. J Thorac Cardiovasc Surg 2004;128:625-6.

20. Kirklin JK, Pagani FD, Kormos RL, et al. Eighth annual INTERMACS report: Special focus on framing the impact of adverse events. J Heart Lung Transplant 2017;36:1080-6.

21. Teuteberg JJ, Cleveland JC Jr, Cowger J, et al. The Society of Thoracic Surgeons Intermacs 2019 Annual Report: The Changing Landscape of Devices and Indications. Ann Thorac Surg 2020;109:649-60.

22. Levin AP, Jaramillo N, Garan AR, et al. Outcomes of contemporary mechanical circulatory support device configurations in patients with severe biventricular failure. J Thorac Cardiovasc Surg 2016;151:530-5.e2.

23. Vierecke J, Gahl B, de By T, et al. Results of primary biventricular support: an analysis of data from the EUROMACS registry. Eur J Cardiothorac Surg 2019;56:1037-45.

24. Shah P, Ha R, Singh R, et al. Multicenter experience with durable biventricular assist devices. J Heart Lung Transplant 2018;37:1093-101.

25. Maltais S, Womack S, Davis ME, et al. Surgical Considerations and Challenges for Bilateral ContinuousFlow Durable Device Implantation. ASAIO J 2016;62:e18-21.
26. Marasco SF, Stornebrink RK, Murphy DA, et al. Long-term right ventricular support with a centrifugal ventricular assist device placed in the right atrium. J Card Surg 2014;29:839-42.

27. Eulert-Grehn JJ, Lanmüller P, Schönrath F, et al. Two implantable continuous-flow ventricular assist devices in a biventricular configuration: technique and results. Interact Cardiovasc Thorac Surg 2018;27:938-42.

28. Krabatsch T, Potapov E, Stepanenko A, et al. Biventricular circulatory support with two miniaturized implantable assist devices. Circulation 2011;124:S179-86.

29. Tran HA, Pollema TL, Silva Enciso J, et al. Durable Biventricular Support Using Right Atrial Placement of the HeartWare HVAD. ASAIO J 2018;64:323-7.

30. Shehab S, Macdonald PS, Keogh AM, et al. Long-term biventricular HeartWare ventricular assist device support-Case series of right atrial and right ventricular implantation outcomes. J Heart Lung Transplant 2016;35:466-73.

31. Marasco S, Simon AR, Tsui S, et al. International experience using a durable, centrifugal-flow ventricular assist device for biventricular support. J Heart Lung Transplant 2020;39:1372-9.

32. Lavee J, Mulzer J, Krabatsch T, et al. An international multicenter experience of biventricular support with HeartMate 3 ventricular assist systems. J Heart Lung Transplant 2018;37:1399-402.

33. McGiffin D, Kure C, McLean J, et al. The results of a single-center experience with HeartMate 3 in a biventricular configuration. J Heart Lung Transplant 2021;40:193-200.

34. Kirklin JK, Naftel DC, Stevenson LW, et al. INTERMACS database for durable devices for circulatory support: first annual report. J Heart Lung Transplant 2008;27:1065-72.

35. Kirklin JK, Naftel DC, Kormos RL, et al. Second INTERMACS annual report: more than 1,000 primary left ventricular assist device implants. J Heart Lung Transplant 2010;29:1-10.

36. Saito S, Toda K, Nakamura T, et al. Rescuing Patients With Severe Biventricular Failure in the Era of Continuous-Flow Left Ventricular Assist Device. Circ J 2019;83:379-85.

37. Fukushima N, Ono M, Saiki Y, et al. Registry Report on Heart Transplantation in Japan (June 2016). Circ J 2017;81:298-303.

38. Schulze PC, Kitada S, Clerkin K, et al. Regional differences in recipient waitlist time and pre- and posttransplant mortality after the 2006 United Network 
for Organ Sharing policy changes in the donor heart allocation algorithm. JACC Heart Fail 2014;2:166-77.

39. Hetzer R, Krabatsch T, Stepanenko A, et al. Long-term biventricular support with the heartware implantable continuous flow pump. J Heart Lung Transplant 2010;29:822-4.

40. Krabatsch T, Hennig E, Stepanenko A, et al. Evaluation of the HeartWare HVAD centrifugal pump for right ventricular assistance in an in vitro model. ASAIO J 2011;57:183-7.

41. Fitzpatrick JR 3rd, Frederick JR, Hiesinger W, et al. Early planned institution of biventricular mechanical circulatory support results in improved outcomes compared with delayed conversion of a left ventricular assist device to a biventricular assist device. J Thorac Cardiovasc Surg

Cite this article as: Farag J, Woldendorp K, McNamara N, Bannon PG, Marasco SF, Loforte A, Potapov EV. Contemporary outcomes of continuous-flow biventricular assist devices. Ann Cardiothorac Surg 2021;10(3):311-328. doi: 10.21037/acs-2021-cfmcs-34
2009;137:971-7.

42. Shehab S, Newton PJ, Allida SM, et al. Biventricular mechanical support devices--clinical perspectives. Expert Rev Med Devices 2016;13:353-65.

43. Bartfay SE, Dellgren G, Lidén H, et al. Are biventricular assist devices underused as a bridge to heart transplantation in patients with a high risk of postimplant right ventricular failure? J Thorac Cardiovasc Surg 2017;153:360-7.e1.

44. Kirklin JK, Naftel DC, Pagani FD, et al. Seventh INTERMACS annual report: 15,000 patients and counting. J Heart Lung Transplant 2015;34:1495-504.

45. Kirsch M, Mazzucotelli JP, Roussel JC, et al. Survival after biventricular mechanical circulatory support: does the type of device matter? J Heart Lung Transplant 2012;31:501-8. 

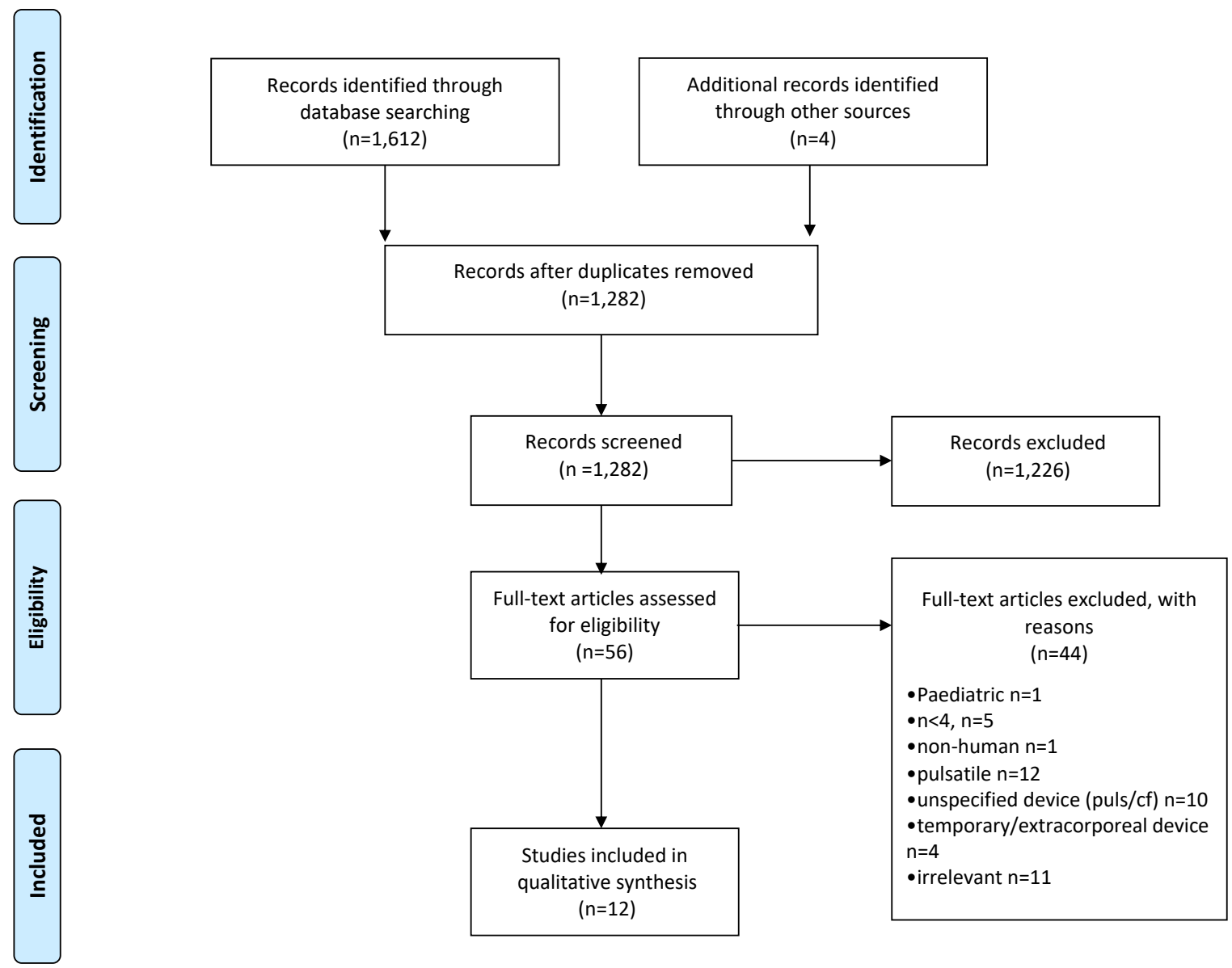

Figure S1 PRISMA flow diagram. cf, continuous flow; puls, pulsatile. 